\title{
ENFOQUES OBJETIVO Y SUBJETIVO DEL CONCEPTO DE INFORMACION
}

\section{J. Carlos Fernández-Molina *}

Resumen: La búsqueda de un concepto adecuado de información ha sido una constante en los estudios teóricos llevados a cabo en el seno de la Documentación. El análisis de las propuestas realizadas permite distinguir dos grandes grupos: las que ven la información como algo físico, objetivo, y las que la consideran como algo cognitivo, subjetivo. Se analizan algunos de los ejemplos más representativos de ambas tendencias, aunque haciendo un especial hincapié en las propuestas del segundo grupo, por considerarlas más relevantes.

Palabras clave: Información, conocimiento, enfoque cognitivo.

\begin{abstract}
The search for an adequate concept of information has been a constant theme in the theoretical studies carried out within the Information Science field. The proposals made can be grouped in two large sets: those that consider information as something physical and objective and those that see it as something cognitive and subjective. Some of the most outstanding examples from both trends are examined. Special attention is paid to the proposals from the second group due to their relevance.
\end{abstract}

Keywords: Information, knowledge, cognitive approach.

\section{Introducción}

Uno de los temas más habituales en los estudios teóricos llevados a cabo en el seno de la Documentación —entendida aquí como sinónimo de "Information Science»- es el concepto de información. Son muy numerosas las definiciones aportadas que intentan distinguirla de otros conceptos similares tales como datos, inteligencia, mensajes, entendimiento, signos o conocimiento, aunque en términos generales tales tentativas han sido poco satisfactorias.

El argumento más utilizado para justificar tales estudios teóricos ha sido la necesidad de que una disciplina que se ocupa del estudio de la información cuente con un concepto consensuado de su objeto de estudio. En este sentido, Maricic (1), basándose en el concepto de paradigma de Kuhn (2), consideraba que la Documentación estaba en situación pre-paradigmática y que un concepto de información era el candidato más apropiado para convertirse en su paradigma.

Sin embargo, encontrar un concepto adecuado de información resulta realmente dificil. En principio, información designa tanto el proceso de comunicar hechos, noticias, como lo que se comunica. Se han descubierto o creado tantas formas de comunicar información y tantas cosas para ser comunicadas (de manera

* Escuela Universitaria de Biblioteconomía y Documentación. Universidad de Granada.

Recibido: 20-5-94. 
mental, acústica, genética, mecánica, eléctrica, etc.) que parece imposible distinguirlas con un solo término. Debons y sus colaboradores (3) ponen de manifiesto este problema haciendo un recorrido por algunas de las formas en que se entiende la palabra información: conocimiento, hechos, datos, artículo de consumo, comunicación, energía, etc. De manera similar, Robert Fairthorne (4) opinaba que el uso de palabras como información es «meramente metafórico, o son etiquetas convenientes para una masa amorfa de actividades y fenómenos mal definidos».

Esta búsqueda podemos plantearla según un enfoque «científico», o sea, un concepto único de información que cumpla una serie de requisitos para ser adecuado para la Documentación, o un enfoque "pragmático", haciendo una simple aproximación a los usos del término información que nos son útiles. Este último planteamiento ha sido utilizado entre otros por Michael Buckland (5), distinguiendo: a) información como proceso: la acción de informar, comunicación del conocimiento...; b) información como conocimiento: lo que se imparte en el proceso informativo, lo que reduce la incertidumbre...; y c) información como cosa: objetos tales como datos o documentos que contienen información, que tienen la cualidad de impartir conocimiento o comunicar información.

Junto a aproximaciones pragmáticas como la anterior, ha habido numerosas propuestas implícitas y explícitas de un concepto de información para la Documentación. Tales propuestas pueden clasificarse en dos grupos fundamentales: las que consideran la información como algo externo, objetivo, tangible, y las que la contemplan como algo subjetivo, cognitivo, situacional. Este trabajo pretende recoger los ejemplos más representativos de ambas tendencias, aunque dedicando una especial atención a las propuestas del segundo grupo, por considerarlas más adecuadas.

\section{La información como algo objetivo}

Durante las primeras décadas de desarrollo de la Information Science -desde 1945 hasta la década de los 60 - la investigación llevada a cabo por los information scientists estaba basada en la metodología propia de las ciencias físicas, por lo que su objetivo primordial consistía en encontrar un concepto de información formalizado, matemático, que se pudiera medir y expresar en unidades físicas. La mayoría de las propuestas tenían su origen en la teoría de Claude Shannon (6), surgida en un contexto de investigación en ingeniería, por lo que se intentó ampliarla para que fuera adecuada al ámbito de estudio global de la Documentación. Más recientemente, además de nuevas propuestas de adaptación de la teoría de Shannon, se han planteado diversas ideas sobre el fenómeno de la información como algo objetivo, de las que creemos que la más interesante es la que la considera como propiedad básica del universo.

\subsection{La Teoría Matemática de la Comunicación}

La Teoría Matemática de la Comunicación de Shannon, con su interpretación a cargo de Warren Weaver (7), dio lugar a la única propuesta formalizada, matemática e implementada de concepto de información. Esto provocó que se aplicara incluso fuera del contexto para el que inicialmente se formuló - como solución 
para un problema de ingenieria-, lo que no deja de ser sorprendente, ya que desde el principio se sabía lo limitados que son los aspectos de los procesos de comunicación que cubre. Es más, según la visión a tres niveles del propio Weaver, paralelos a los tres niveles semióticos de Morris (sintáctico, semántico y pragmático), la teoría de Shannon sólo era una teoría sintáctica, por lo que no podía ser utilizada para una ciencia en la que el elemento semántico es fundamental (8).

La teoría de Shannon define la noción de "contenido de información" de un tipo de acontecimiento concreto: la recepción de una señal que ha sido transmitida sobre una línea de transmisión. Estas señales no son entendidas necesariamente como teniendo un tipo concreto de significado, sino que la información se define sólo en términos de la probabilidad de recepción de las diferentes señales. De esta forma, la información de una señal poco probable es alta y la de una señal probable es baja. La definición formal de «información» es el logaritmo de la reciproca de la probabilidad de la señal. Por tanto, cuando la probabilidad es cercana a cero, su recíproca es grande y también lo será su información (el valor del logaritmo). Por el contrario, si la probabilidad es cercana a uno, su recíproca es pequeña, y el logaritmo cercano a cero, al igual que su carga informativa (9).

Aunque el propio Shannon dejó bien claro que los «aspectos semánticos de la comunicación son irrelevantes desde la perspectiva de la ingeniería..., lo importante es que el mensaje se selecciona de un conjunto de posibles mensajes» (7), han sido muy numerosos los intentos de utilizar su teoría en el contexto global de la Documentación - aunque sin demasiado éxito-, entre los que se pueden destacar los de Susan Artandi (10) y Jack Belzer (11). No obstante, quizá sin pretenderlo, el propio Shannon también contribuyó a la confusión al publicar en 1951 un trabajo en el que trataba de información con significado. Planteó que en lugar de un código para cada letra del alfabeto inglés, ¿por qué no tener un código de segundo orden para digrams (o sea, para pares de letras tales como "th», «ed», etc.) con longitudes de código inversamente proporcionales a su frecuencia de ocurrencia? $O$ ir más allá y tener códigos de tercer orden o trigrams (12). Esta idea ha sido aplicada más tarde en la práctica.

En la actualidad, sigue abierta la cuestión de si la teoría de Shannon puede ser significativa no sólo a nivel sintáctico, sino también aplicada a estudios semánticos y pragmáticos de procesos de información. Muy recientemente, Charles Cole (13) ha propuesto una nueva interpretación, en la que sugiere que en la teoría de Shannon se pueden encontrar dos distintos conceptos de información, de incertidumbre y de entropía. Considera que la interpretación tradicional se ha centrado más en la incertidumbre asociada con la transmisión de la señal - punto de vista del receptor-, que en la asociada con la selección de un mensaje del conjunto de posibles mensajes - punto de vista del emisor-. En su opinión, esta última perspectiva es mucho más apropiada para ser utilizada en el contexto global de la Documentación.

\subsection{Información como propiedad básica del universo}

Tom Stonier $(14,15)$ plantea la necesidad de una nueva teoría de la información, cuyo presupuesto básico es que la información es una propiedad básica del 
universo, como la materia y la energía. Como ésta tiene realidad física y existe en diversas formas que se transforman e intercambian.

Teniendo en cuenta que cualquier sistema que tiene organización contiene información, Stonier expresa su extrañeza por el poco interés que ha despertado el estudio científico de la información hasta hace pocos años. En su opinión, esto se debe, por un lado, a que la rápida intertransformación de información y energía hacía posible siempre interpretar los cambios de estado de nuestro universo en términos de energía y, por otro, a que sólo muy recientemente se ha visto la necesidad de conceptualizarla y cuantificarla de manera independiente, especialmente por parte de ingenieros preocupados por las comunicaciones o de biólogos interesados en el ADN. Serán los ordenadores los que cambien esta situación e impliquen a más personas en el estudio de la información.

La información, que existe independientemente de que cualquier humano u otra forma de inteligencia la perciba o la utilice, puede ser de dos tipos: estructural y cinética. Información estructural será la contenida en un sistema, por ejemplo en un libro, en una molécula de $\mathrm{ADN}$, en un átomo. Información cinética es la que se transmite, procesa o transforma, por ejemplo cuando se lee un libro o cuando una molécula de ADN es procesada por una célula. Ambos tipos de información pueden interconvertirse mutuamente sin ningún problema.

Basado en estos presupuestos, Stonier afirma que una verdadera teoría general de la información debería ser capaz de definir con absoluta precisión y claridad términos como información, inteligencia, significado y entendimiento. Debería además explicar y cuantificar claramente estos fenómenos, de manera que pudiéramos contestar a cuestiones prácticas, tales como cuánta información tiene un programa de ordenador o cuánta información estamos obteniendo al leer un libro.

Esta aportación de Stonier representa la visión más global de entendimiento de la información, en la que se mezclan lo que para otros son datos, conocimiento, fuerzas, influencias, etc. En la actualidad, la mayoría de los documentalistas consideran excesivo este concepto tan omnicomprensivo y ciertamente difuso de información. No obstante, parece un buen intento de ampliar el entendimiento del fenómeno de la información, que puede abrir la posibilidad de nuevas ideas que ayuden al desarrollo de una teoría de la información.

\section{La información como algo subjetivo}

A finales de los años 70 y principios de los 80 vieron la luz diversos trabajos cuya premisa básica era la crítica contra el enfoque tradicional con el que se habían estudiado las necesidades, el uso y el propio concepto de información. Autores como Belkin (16), Wilson (17) o Dervin (18) coincidian en poner en duda la validez y utilidad de la labor investigadora llevada a cabo en este campo y proponian enfoques alternativos. La mayoría de estos autores estaban de acuerdo en que los estudios hechos hasta ese momento se centraban en un concepto de información como algo objetivo, externo, con un significado constante (19). Sin duda, este afán por la "objetividad" tenía su origen en que la mayoria de los information scientists provenían de las ciencias exactas o aplicadas e introdujeron sus métodos y hábitos en el estudio de la información, dejando a un lado sus aspectos humanos. Sin 
embargo, en los procesos de transferencia de información, como en cualquier fenómeno social, intervienen factores de carácter subjetivo, tales como las creencias, valores, lenguaje, intuición, imaginación, etc., que no son susceptibles de adaptarse a las exigencias del método científico (20).

De esta forma, proponen que se utilice un nuevo enfoque en el estudio de la información en el seno de la Documentación, que ha sido denominado generalmente como punto de vista o enfoque cognitivo. Su premisa básica es que en ambos extremos de un sistema de comunicación (emisor y receptor) se producen procesos cognitivos.

Dado que el término "cognitivo» se viene aplicando en contextos muy diferentes y con distintos sentidos, es conveniente que fijemos de manera explícita el significado con que nosotros lo utilizamos. Una definición de este enfoque, que se ha convertido ya en clásica, fue la que dio Marc De Mey en el congreso internacional específico sobre este tema celebrado en 1977: se basa en que «cualquier procesamiento de información, ya sea perceptual o simbólico, es mediado por un sistema de categorías o conceptos que, para el dispositivo de procesamiento de la información, son un modelo de su mundo» (21).

A continuación vamos a analizar las aportaciones teóricas más desarrolladas y representativas de dicho enfoque o punto de vista.

\subsection{Información como acontecimiento}

Allan Pratt (22) ha intentado determinar un concepto de información específico para la Documentación, desarrollando un modelo de sistema de comunicación del que extrae un significado de información. Según su enfoque, información es un acontecimiento que tiene lugar en una etapa concreta del proceso de comunicación.

Su modelo de comunicación consta de dos sistemas separados: uno compuesto por emisor, propósito, lenguaje y medio, que da como resultado un registro gráfico; el otro de receptor, predisposición y conocimiento de -o preferencia por - lenguaje y medio. El medio para la comunicación entre emisor y receptor es el emparejamiento de predisposición con propósito, y lenguaje y medio del receptor con lenguaje y medio del emisor (o sea, con el registro gráfico que el emisor ha producido).

Para simplificar, tanto el emisor como el receptor pueden ser representados por sus «imágenes» (en el sentido que le da a este término Kenneth Boulding (23): la concepción mental que tenemos de nuestro entorno y de nosotros mismos en él). En cuanto a los componentes de propósito y predisposición del sistema de comunicación, se corresponden con el intento de cambiar una imagen del receptor y con el consentimiento o deseo de que esa imagen sea cambiada, respectivamente. Dentro de este contexto, «información es la alteración de la imagen que ocurre cuando se recibe un mensaje» (22).

Para Pratt el acontecimiento clave en un sistema de comunicación es lo que ocurre dentro de la mente con la absorción de un mensaje. Opina que información es el término adecuado para ese acontecimiento, esto es, información es el acontecimiento efectivo, que produce un efecto. 


\subsection{Información como estructura}

Nicholas Belkin se ha dedicado con bastante entusiasmo a desarrollar un concepto de información específico para la Documentación, esfuerzo que se plasma fundamentalmente en su tesis (24). Antes de ésta, en colaboración con Stephen Robertson, ya había hecho una primera aproximación.

Consideran que la única noción básica común a todos los usos de información es la idea de que cambia las estructuras, por lo que sugieren que información es «lo que es capaz de transformar la estructura». Además de la idea de estructura, utilizan la de «texto» para alcanzar su definición de información. Texto será «una colección de signos estructurados de manera deliberada por un emisor con la intención de cambiar la estructura de la imagen de un receptor», por lo que información es «la estructura de cualquier texto que es capaz de cambiar la estructura de la imagen de algún receptor» (25).

Algo más tarde, Belkin extiende y modifica este concepto, especialmente a través de su idea de "estado anómalo de conocimiento» (ASK) del receptor, que sería lo que instigaría el funcionamiento del sistema de comunicación.

El sistema se resume de la forma siguiente: un emisor potencial de un texto decide comunicar algún aspecto de su estado de conocimiento sobre el mundo. Esta decisión afecta a una parte concreta de todo su estado de conocimiento, que deberá ser aislada. Esta constituiría el fundamento de la estructura conceptual del emisor, que es la base de lo que va a ser comunicado. Pero antes de que esa estructura sea comunicable sufrirá varias transformaciones de acuerdo con su propósito e intención y con el conocimiento de los receptores proyectados. Es decir, estos tres factores producen que la estructura conceptual del emisor adopte diversas formas. Esta estructura modificada se convertirá por medio del lenguaje u otro medio en una estructura comunicable, el texto, que pasará a ser uno de los posibles textos a que los receptores potenciales tendrán acceso. Serán éstos los que instiguen la puesta en marcha del sistema de comunicación, al reconocer una anomalia en su estado de conocimiento, que deberá convertirse en una estructura comunicable (una pregunta, por ejemplo). Ambas estructuras interactuarán y será el receptor el que decida si la anomalia ha sido resuelta, en cuyo caso el sistema se cerraría, o no lo ha sido, con lo que el sistema volvería a ser instigado (16).

En este sistema de comunicación hay un elemento que puede servir como concepto de información:

"la información asociada con un texto es la estructura conceptual modificada del emisor (por el propósito, intención y conocimiento del estado de conoci-

miento del receptor) que sirve de base a la estructura superficial (por ejemplo el lenguaje) de ese texto" (24).

Este concepto se basa en la combinación de un sistema de comunicación cognitivo, una representación estructural del conocimiento y la instigación del receptor vía estado anómalo de conocimiento.

\subsection{Brookes y su ecuación fundamental}

Bertram Brookes ha sido uno de los primeros en insistir en la importancia del punto de vista cognitivo en la Documentación: «el fenómeno de mayor interés para 
la documentación son las interacciones cognitivas entre usuarios y los sistemas públicos de conocimiento organizados para facilitar la labor de referencia al usuario en bibliotecas y bases de datos» (26).

Brookes propuso en 1974 su «ecuación fundamental» para la Documentación, que nos ofrece de manera implícita un concepto de información. La ecuación ha sufrido pequeñas modificaciones hasta presentar la forma siguiente (27):

$$
K[S]+\Delta I=K[S+\Delta S]
$$

Esta ecuación expresa de manera general que la estructura de conocimiento $(K[S])$ es cambiada a una nueva estructura modificada $(K[S+\Delta S])$ por la información $(\Delta I)$; el efecto de la modificación es indicado por $\Delta S$. Por tanto, nos ofrece, aunque de manera implícita, una definición de información como «eso» que modifica la estructura de conocimiento.

La ecuación está expresada en forma pseudo-matemática (los términos y simbolos no están realmente definidos) por considerar su autor que es la forma más compacta en que la idea puede ser expresada. No obstante, muestra de manera implícita que si sus entidades son medibles, deberían ser medidas en las mismas unidades, esto es, que información y conocimiento pertenecen a la misma clase y tienen las mismas dimensiones. Sin embargo, no pueden ser sustituidas en la ecuación para indicar que un mismo $\Delta I$ puede producir distintos efectos sobre diferentes estructuras de conocimiento, o sea, que hay un importante componente de subjetividad.

De esta forma, Brookes considera "conocimiento como una estructura de conceptos unidos por sus relaciones, e información como una pequeña parte de tal estructura. La estructura de conocimiento puede ser subjetiva u objetiva» (27). Esta última consideración está directamente relacionada con la idea de Brookes de identificar el campo de la Documentación con el «Mundo 3» de Popper (28), el del conocimiento objetivo, por lo que admite la posibilidad de estructuras de conocimiento objetivas, esto es, registradas.

Este concepto de información está directamente relacionado con el de Belkin en la idea de información como estructura, pero muestra un mayor énfasis sobre la posibilidad de ser medida de manera objetiva.

\subsection{El concepto de síntesis de Ingwersen}

Peter Ingwersen ha propuesto recientemente un concepto de información específico para el contexto de la Documentación. Está basado fundamentalmente en el concepto de Belkin y en la ecuación fundamental de Brookes, que son extendidos en un intento de fusionar y completar ambas aportaciones.

Como punto de partida utiliza una versión de la ecuación fundamental, que él considera más dinámica (29):

$$
\Delta I+K[S] \rightarrow K[S+\Delta S]
$$

Ingwersen explica el proceso que se produce de la siguiente forma. Cuando sccedemos al «Mundo 3» de Popper, el del conocimiento objetivo (si abrimos un 
libro, por ejemplo), nos encontramos sólo con datos, que se comunican mediante signos, símbolos, palabras, texto, etc. Con la percepción, los datos son transformados por la actual estructura de conocimiento en información, omitiendo datos de sobra que no se perciben. Los conceptos y sus relaciones se reconocen y se almacenan en la memoria. Esta es la nueva información que puede transformar la estructura de conocimiento. La forma en que la nueva información afecta a la estructura de conocimiento dependerá de su estado de conocimiento y de la complejidad de la información percibida.

De este esquema Ingwersen extrae varias conclusiones: 1) desde el punto de vista del receptor la información es algo potencial hasta que se recibe; 2) desde el punto de vista del emisor los receptores también son potenciales; 3) cuando en un «estado de incertidumbre» un receptor accede a la información potencial, convierte datos, que pueden convertirse en información sólo si se perciben; 4) si no se percibe la información potencial, permanecerá como datos para ese receptor concreto y como información potencial para otros receptores y emisores; 5) la percepción es controlada por la estructura de conocimiento actual en el estado de conocimiento y espacio-problema actuales; 6) la información $(\Delta I)$ puede servir de apoyo al estado de incertidumbre y transformar el espacio-problema y el estado de conocimiento, causando decisiones, acciones, intenciones, cambio de valores, etc., o sea, producir un efecto; 7) la información es una transformación de estructuras de conocimiento (30).

Basado en estas ideas, propone una modificación y ampliación de la ecuaciór de Brookes, que incluye la generación de información (31):

$$
p I \rightarrow \Delta I+K[S] \rightarrow K[S+\Delta S] \rightarrow p I^{\prime}
$$

En este modelo, de la información potencial $(p I)$ se percibe sólo una parte $(\Delta I)$. que interactúa con el estado de conocimiento actual $(K[S])$ (incluyendo el espacio problema y el estado de incertidumbre), transformándolo en uno nuevo $(K[S+\Delta S])$ con un efecto $(\Delta S)$. El estado de conocimiento modificado puede generar nueva información $\left(p I^{\prime}\right)$, potencial para otros receptores.

De este modelo se deduce que información es «el resultado de una transformación de estructuras de conocimiento del emisor (por la intencionalidad, el modelo de estados de conocimiento de receptores y en forma de signos)» y que «es algo (una estructura) que cuando se percibe puede afectar y transformar el estado de conocimiento del receptor" (30).

Por tanto, la información sólo tiene lugar en la comunicación humana y siempre que la información potencial sea procesada por el emisor, así como por el receptor. En el momento en que la información transforme el estado de conocimiento del receptor se convertirá en conocimiento.

Por otro lado, ya que es el receptor el que instiga la comunicación, sin él los sistemas de información sólo son «sistemas de datos» o "sistemas de información potencial». Una vez que se les interroga, la información potencial o datos se transmite por un canal y en el momento de ser percibida por el receptor se convierte en información. Mediante la integración con el estado de conocimiento actual a través del espacio-problema y resolviendo el estado de incertidumbre del receptor, la información se convierte en conocimiento. En suma, constantemente 
estamos limitados al nivel lingüistico de la comunicación, operando sólo con datos o información potencial en forma de signos, texto, imagen, etc., de manera que únicamente en el momento de transformación del estado de conocimiento de un receptor la comunicación tiene lugar a un nivel cognitivo.

En consecuencia, los sistemas de información basados en el ordenador no procesan información en este sentido, sino datos o información potencial. Sólo los humanos son capaces de procesar información a un nivel cognitivo. No obstante, la Documentación no sólo se ocupa del estudio de la información en este sentido restringido, sino que se dedica a la mejora de los métodos de organización y acceso intelectual a la información potencial almacenada para facilitar y apoyar su utilidad para los receptores potenciales humanos (30).

En este esquema conceptual encontramos aportaciones muy interesantes, ya apuntadas por otros autores. En especial, se establece una clara distinción y relación entre datos, información y conocimiento: los datos son información potencial, que sólo si se perciben por el receptor se convierten en información, y ésta pasa a convertirse en conocimiento en el momento en que produce una modificación de la estructura de conocimiento de un receptor.

\subsection{Teoría de «sense making»}

Una de las aportaciones teóricas que más impacto ha causado y más apoyos ha recibido en la comunidad científica es la teoría de sense making - se podría traducir como «teoría de dar sentido»-, desarrollada desde finales de los 70 por Brenda Dervin y sus colaboradores. Su punto de partida es que el enfoque tradicional con el que se han estudiado las necesidades y el uso de información, centrado en el intermediario y en la institución, debe ser abandonado por un enfoque más subjetivista, centrado en el usuario (19). Sugiere nuevos planteamientos teóricos y metodológicos que revelan la importancia de los factores cognitivos y subjetivos del fenómeno de la información (32).

El objetivo principal de esta teoría es «el comportamiento, tanto interno como externo, que permite al individuo construir y diseñar su movimiento a través del espacio y el tiempo» (33). El comportamiento de «dar sentido» es por tanto un comportamiento comunicativo, es un proceso cuyo resultado es el «sentido». Según Dervin, «sentido» incluye el conocimiento y además el conjunto de factores subjetivos que reflejan las interpretaciones del individuo de una situación, incluyendo opiniones, intuiciones, corazonadas, valoraciones, preguntas, etc.

Aunque el objetivo de esta teoría no es aportar un concepto de información, sino establecer un nuevo modelo de la búsqueda de información - concretamente un modelo constructivista-, es posible deducir de ella una interesante conceptualización de la información. Para Dervin la información no es algo objetivo y externo, sino algo construido por el usuario. El mundo en el que nos movemos está cambiando constantemente, por lo que tenemos que crear el «sentido» de nosotros mismos y de nuestro entorno, para lo que construimos mapas cognitivos de nuestro entorno que se van cambiando y refinando conforme vamos recibiendo nueva información. Esto es, no nos adaptamos a un mundo estático, sino que creamos una realidad que cambia con nosotros (34). 
En definitiva, considera que la información debe ser conceptualizada como el sentido creado en un momento específico del espacio-tiempo por uno o más humanos. Por tanto, la información es subjetiva, no es algo que exista aparte del comportamiento humano, sino que es una construcción personal creada por observadores humanos (35).

\section{Conclusiones}

Las aportaciones analizadas en el apartado anterior tienen como presupuesto común la idea de información como algo subjetivo, individualizado, que forma parte del proceso continuo que sigue cualquier persona en su relación con el entorno que la rodea.

Además, son una representación significativa de la fuerte tendencia, observable en los últimos años, a que el estudio del fenómeno informacional se realice mediante enfoques centrados en el usuario, en el individuo, en detrimento de los centrados en el intermediario, como había sido tradicional. Aunque tales aportaciones no son totalmente convergentes y contienen algunas discrepancias entre ellas, todas comparten la idea de lo limitado que resulta el enfoque clásico centrado en el intermediario y en la idea de información como un objeto físico, por lo que reclaman un cambio de paradigma.

El argumento común que une a todas estas contribuciones teóricas es la importancia que se les reconoce a los factores cognitivos en la transferencia de la información, por lo que todas ellas pueden incluirse en el denominado enfoque o punto de vista cognitivo. En este sentido, señala Bernd Frohmann (36) que este enfoque se presenta a sí mismo no como una teoría entre otras muchas, ni como una teoría sectorial dedicada a problemas específicos, sino como la única teoría global para la Documentación.

No obstante, aunque es evidente que los estudios cognitivos ofrecen unas grandes posibilidades para el desarrollo futuro de la Documentación y ya han comenzado a ser utilizados y validados en estudios empíricos - en especial la teoría de sense making (37)-, hay que reconocer que todavía estamos muy lejos de alcanzar el denominado "paradigma cognitivo". Incluso hay autores que han llamado la atención sobre el excesivo optimismo que ha generado esta perspectiva. Entre ellos, Brian Vickery (38), refiriéndose específicamente al área de recuperación de la información, pone en duda la existencia de tal paradigma cognitivo y, aun admitiendo su presencia, considera que pasará bastante tiempo antes de que sea realmente utilizado en el diseño de sistemas de recuperación de la información.

En definitiva, consideramos que el enfoque cognitivo, aunque todavía no suficientemente desarrollado, parece aportar soluciones válidas para la conceptualización de la información y, en general, para servir como medio de integración y relación a las distintas áreas de estudio que conforman la documentación.

\section{Referencias}

1. MARICIC, S. Information science as interfaces of the cognitive sphere and society. Information Processing and Management, 1987, 23(1), 33-43. 
2. KUHN, T. S. La estructura de las revoluciones cientificas. México D.F.: Fondo de Cultura Económica, 1989.

3. DEBONS, A.; HORNE, E., y CRONENWETH, S. Information science: an integrated view. Boston: G. K. Hall, 1988.

4. FAIRTHORNE, R. A. The morphology of «information flow». Journal of the Association for Computing Machinery, 1967, 14(4), 710-719.

5. BUCKLAND, M. K. Information and information systems. New York: Greenwood Press, 1991.

6. SHANNON, C. E. A mathematical theory of communication. Bell System Technical Journal, 1948, 27, 379-423, 623-656.

7. SHANNON, C. E., y WEAVER, W. Teoria matemática de la comunicación. Madrid: Forja, 1981.

8. SUPPE, F. R. Toward an adequate information science. En Heilprin, L. B., ed. Toward foundations of information science. White Plains, NY: Knowledge Industry Publications, 1985, 7-27.

9. BARNES, R. F. Instead of Shannon, what? En Heilprin, L. B., ed. Toward foundations of information science. White Plains, NY: Knowledge Industry Publications, 1985, 29-34.

10. ARTANDI, S. Information concepts and their utility. Journal of the American Society for Information Science, 1973, 24(4), 242-245.

11. BELZER, J. Information theory as a measure of information content. Journal of the American Society for Information Science, 1973, 24(5), 300-304.

12. SHANNON, C. E. Prediction and entropy of printed english. Bell System Technical Journal, 1951, 30(1), 50-64.

13. COLE, C. Shannon revisited: information in terms of uncertainty. Journal of the American Society for Information Science, 1993, 44(4), 204-211.

14. STONIER, T. Towards a new theory of information. Journal of Information Science, 1991, 17(5), 257-263.

15. STONIER, T. Information and the internal structure of the universe. London: Springer, 1990.

16. BELKIN, N. J. Information concepts for information science. Journal of Documentation, 1978, 34(1), 55-85.

17. WILSON, T. D. On user studies and information needs. Journal of Documentation, 1981, 37(1), 3-15.

18. DERVIN, B. Information as user construct: the relevance of perceived information needs to synthesis and interpretation. En Ward, S. A., y Reed, L. J., eds. Knowledge structures and use: implications for synthesis and interpretation. Philadelphia: Temple University Press, 1983, 153-184.

19. DERVIN, B., y NILAN, M. Information needs and uses. Annual Review of Information Science and Technology, 1986, 21, 3-33.

20. NEILL, S. D. Dilemmas in the study of information: exploring the boundaries of information science. New York: Greenwood Press, 1992.

21. DE MEY, M. The cognitive viewpoint: its development and its scope. En De Mey, M., ed. International Workshop on the Cognitive Viewpoint. Ghent: University of Ghent, 1977, xvi-xxxii.

22. PRATT, A. D. The information of the image. Libri, 1977, 27(3), 204-220.

23. BOULDING, K. The image. Ann Arbor, MI: University of Michigan Press, 1956.

24. BELKIN, N. J. A concept of information for information science. Ph. D. Thesis, University of London, 1977.

25. BELKIN, N. J., y ROBERTSON, S. E. Information science and the phenomenon of information. Journal of the American Society for Information Science, 1976, 27(4), 197-204. 
26. BROOKES, B. C. Measurement in information science: objective and subjective metrical space. Journal of the American Society for Information Science, 1980, 31(4), 248-255.

27. BROOKES, B. C. The foundations of information science. Part I. Philosophical aspects. Journal of Information Science, 1980, 2(3-4), 125-133.

28. POPPER, K. R. Conocimiento objetivo: un enfoque evolucionista. 3. ${ }^{\mathrm{a}}$ ed. Madrid: Tecnos, 1988.

29. BROOKES, B. C. The developing cognitive viewpoint in information science. En De Mey, M., ed. International Workshop on the Cognitive Viewpoint. Ghent: University of Ghent, 1977, 195-203.

30. INGWERSEN, P. Information and information science in context. Libri, 1992, 42(2), 99-135.

31. INGWERSEN, P. A cognitive view of three selected online search facilities. Online Review, 1984, 8(5), 465-492.

32. SAVOLAINEN, R. The sense-making theory: reviewing the interests of a user-centered approach to information seeking and use. Information Processing and Management, 1993, 29(1), 13-28.

33. DER VIN, B. From the mind's eye of the «user»: the sense-making qualitative-quantitative methodology. En Glazier, J. D., y Powell, R. R., eds. Qualitative research in information management. Englewood, CO: Libraries Unlimited, 1992, 61-84.

34. MORRIS, R. C. T. Toward a user-centered information service. Journal of the American Society for Information Science, 1994, 45(1), 20-30.

35. DERVIN, B. Audience as listener and learner, teacher and confidant. The sensemaking approach. En Rice, R., y Atkins, C., eds. Public communication campaigns. Newbury Park, CA: Sage Publications, 1989, 67-86.

36. FROHMANN, B. The power of images: a discourse analysis of the cognitive viewpoint. Journal of Documentation, 1992, 48(4), 365-386.

37. JACOBSON, T. L. Sense-making in a database environment. Information Processing and Management, 1991, 27(6), 647-657.

38. VICKERY, B. C. Knowledge structures in information retrieval. Journal of Documentation, 1992, 48(3), 326-328. 